\title{
Émergence des plantules de carotte (Daucus carotta $L$ ) sous des obstacles mécaniques superficiels
}

\author{
V Tamet ${ }^{1}, \mathrm{~N}$ Souty ${ }^{2 \star}, \mathrm{C}$ Rode ${ }^{2}$ \\ 1 INRA, unité d'agronomie, rue Fernand-Christ, F02007 Laon cedex, France \\ 2 INRA, unité de science du sol, centre de recherches d'Avignon, domaine Saint-Paul, site Agroparc, \\ F84914 Avignon cedex 9, France
}

(Reçu le 15 juillet 1994; accepté le 9 mai 1995)

\begin{abstract}
Résumé - La phase d'implantation constitue une étape cruciale et difficile du cycle de culture de la carotte. Il importe de déceler les causes des faibles taux de levée et de l'irrégularité du peuplement végétal fréquemment observés dans la pratique et qui sont à l'origine de récoltes très hétérogènes. Les études entreprises en laboratoire ont porté sur l'effet de facteurs variant de façon importante dans les conditions d'implantation de la culture : poids de semences, profondeur de semis, température, responsables de la variation de la force de croissance de la plantule que nous avons mesurée à l'aide d'un dispositif mis au point au laboratoire. Ces forces, en moyenne inférieures à $10 \mathrm{~g}(0,1 \mathrm{~N})$, sont d'une part faibles par comparaison avec d'autres espèces végétales, ce qui peut expliquer l'importante sensibilité des plantules aux obstacles mécaniques superficiels. D'autre part, ces forces présentent une grande variabilité même en considérant des plantules issues d'une même classe de poids de semences et placées dans des conditions de milieu identiques, ce qui peut expliquer l'hétérogénéité d'un peuplement. Par ailleurs le taux d'émergence de plantules confrontées à des croûtes superficielles artificielles a été suivi et déterminé en fonction des propriétés mécaniques de ces obstacles. L'établissement d'une relation entre les caractéristiques des obstacles mécaniques et la force des plantules à l'aide de la théorie de la flexion des plaques circulaires a permis d'élaborer un modèle d'émergence.
\end{abstract}

Daucus carotta $\mathrm{L}=$ carotte $/$ poids des semences / force de croissance / profondeur de semis / croûtes de battance / modèle d'émergence

Summary - Emergence of carrot seedlings (Daucus carotta $\mathrm{L}$ ) from under artificial obstacles. Carrot crop establishment is difficult and the percentage of emergence varies widely with soil properties, seedling environmental factors and seedling characteristics. This study took into account seed weight, sowing depth and seed-bed temperature, which influence the growth force of the seedling. The growth forces were measured in the laboratory (about $10 \mathrm{~g}$ or $0.1 \mathrm{~N}$ ). They were lower than the growth forces of other species and very variable for the same experimental conditions. The effect of the surface soil layer on the percentage of emergence was also studied. The theory of bending circular brittle plates allowed us to obtain a relationship between the characteristics of the obstacles and growth forces, which provided the possibility of designing an emergence model.

\section{seed weight / growth force / sowing depth / crust / emergence model}




\section{INTRODUCTION}

La production française de carottes, relativement stable au cours de ces 10 dernières années (la carotte est le deuxième légume consommé en France), est actuellement confrontée à des problèmes de précocité de mise sur le marché et de qualité du produit commercialisé. Pour l'avenir de cette culture, la maîtrise de ces 2 aspects est capitale.

Les normes de qualité, portant en particulier sur les dimensions de la racine, définissent le poids moyen recherché pour une racine, déterminant ainsi le rendement net (nombre de plantules dans la norme $x$ poids moyen d'une racine dans la norme). Ce rendement est soumis à des exigences commerciales strictes, en particulier pour le calibre caractérisé par le diamètre : 8 à $20 \mathrm{~mm}$ pour la petite carotte pour l'appertisation, 25 à $45 \mathrm{~mm}$ pour la carotte de frais et 5 à $6 \mathrm{~cm}$ pour la grosse carotte.

Le calibre dépend, d'une part, de la densité de peuplement (250 à 350 carottes au $\mathrm{m}^{2}$ pour les petites carottes de l'industrie et 70 à 80 carottes au $\mathrm{m}^{2}$ pour les carottes de frais) et d'autre part de l'homogénéité du peuplement à l'issue de la phase d'implantation de la culture (Salter et al, 1980 et 1981 ; Benjamin, 1982 et 1987).

La date de récolte (c'est-à-dire de la précocité de mise sur le marché) est liée à la date de semis et à la durée nécessaire au cycle de la culture en fonction des conditions de milieu. La densité de peuplement dépend des modalités de semis et des conditions de milieu rencontrées au cours de la phase d'implantation. Ainsi les déterminants agronomiques de la date de récolte et du poids moyen de la racine peuvent être principalement rattachés à la phase d'implantation de la culture.

Or l'implantation est en général mal maîtrisée et présente une forte variabilité de réussite (Villeneuve et Leteinturier, 1992). Elle doit souvent se réaliser en conditions qui peuvent être considérées comme défavorables et qui se traduisent par des taux de levée faible et une levée étalée. En effet les cultures de carottes sont généralement menées sur des sols de texture légère permettant un enracinement régulier et facilitant l'arrachage à la récolte (Nicoullaud, 1988) mais très sensibles à la battance. Par ailleurs pour les semis réalisés le plus souvent au printemps, une température parfois basse peut ralentir la croissance et prolonger la phase d'implantation.
Une étude bibliographique exhaustive des problèmes d'émergence en général a d'ailleurs été réalisée par Goyal (1982) et il en ressort que l'effet néfaste des croûtes peut être réduit entre autres par le choix de semences capables d'exercer des forces importantes. Les facteurs et les processus responsables d'une faible force, donc d'une mauvaise implantation, sont supposés fortement déterminés par les modalités de redistribution des réserves de la semence vers la plantule et ses organes. Les travaux de Durr et al (1990) et de Tamet (1992) ont permis d'expliciter ces relations et d'examiner comment elles sont modulées par différents facteurs susceptibles de présenter une gamme de variation importante : poids des semences, profondeur de semis, température, résistance mécanique des horizons de surface. Ces études ont conduit à distinguer 3 phases de croissance souterraine des plantules de carottes. La première phase (de 0 à $4 \mathrm{j}$ après la germination, à $20^{\circ} \mathrm{C}$ ) est une phase de transfert des réserves séminales vers la plantule via les cotylédons qui se dégagent peu à peu du résidu de la semence. La plantule se détache alors de la semence et ne croît plus aux dépens des réserves. La deuxième phase (entre 4 et $8 j$ après la germination à $20^{\circ} \mathrm{C}$ ) correspond à une redistribution pondérale au sein de la plantule, essentiellement des cotylédons vers l'hypocotyle qui continue à s'allonger. La troisième phase (audelà de $8 \mathrm{j}$ après la germination à $20^{\circ} \mathrm{C}$ ) est caractérisée par l'arrêt de l'augmentation des poids et longueurs de tous les organes. L'évolution morphologique de l'hypocotyle au cours de la croissance souterraine dépend essentiellement de la durée de la croissance souterraine, de la masse des semences et de la température.

L'objectif du travail présenté est de dégager l'influence, d'une part, de ces 3 facteurs sur les caractéristiques mécaniques de l'hypocotyle et, d'autre part, des propriétés des sols sur les possibilités d'émergence des plantules quand elles sont confrontées à un obstacle mécanique superficiel. De plus nous essaierons d'établir une corrélation entre caractéristiques de la plantule et caractéristiques du sol afin de pouvoir élaborer un modèle d'émergence.

\section{MATÉRIELS ET MÉTHODES}

Pour réaliser cette étude 2 sortes d'expérimentations en laboratoire ont été menées : des mesures de forces de croissance de plantules et des suivis d'émergence de plantules à travers des croûtes superficielles. II était donc nécessaire de posséder un 
matériel végétal bien caractérisé, de fabriquer des croûtes de caractéristiques bien déterminées et reproductibles et de réaliser des dispositifs expérimentaux fiables.

\section{Matériels}

\section{Matériel végétal}

\section{Semences}

Comme dans les travaux de Durr et al (1990) et de Tamet (1992), les semences appartiennent à la variété Nandor (Clause, type Nantaise améliorée) qui est une variété hybride $\mathrm{F} 1$; elle a été choisie parmi d'autres à cause de sa germination rapide et homogène fournissant un taux final de germination élevé (environ $96 \%$ ). Les lots de semences de cette variété sont vendus sous plusieurs calibres, issus de tris sur la densité, la longueur et la largeur des semences. Dans le calibre choisi, 1,6-1,8 mm, 2 classes de poids de semences ont été retenues : 0,5 à $1,0 \mathrm{mg}$ (petites semences : PS) et 1,6 à $2,2 \mathrm{mg}$ (grosses semences : GS).

\section{Germination}

Les semences sont mises à germer sur buvards, selon les normes des tests ISTA (1985), et placées dans une enceinte climatique. Pour réduire la variabilité liée à l'hétérogénéité des dates de germination des semences, nous avons sélectionné, pour les expérimentations, les plantules issues de semences à dates de germination voisines (la date de germination est connue individuellement avec une précision de $3 \mathrm{~h}$ ). Ainsi à $20^{\circ} \mathrm{C}$ nous avons choisi les plantules provenant de semences ayant germé entre 2,4 et $3 j$ après le semis et à $10^{\circ} \mathrm{C}$ les plantules issues de semences ayant germé entre 5,2 et 6,3 j après le semis. Les plantules sont alors prêtes pour être repiquées.

\section{Obstacles superficiels : croûtes de sol}

Elles sont fabriquées au laboratoire puis placées à la surface d'un pot de culture rempli de sable. Pour qu'elles soient homogènes, continues et isotropes, nous avons préparé une pâte saturée (rendue homogène par agitation) à partir d'un sol tamisé à $2 \mathrm{~mm}$ de granulométrie : argile : $9,1 \%$, limons : $53,4 \%$ et sables : $33,3 \%$. C'est un sol de la Manche (région de cultures de carottes). De plus puisque au champ la zone entourant la plantule peut être assimilée mécaniquement à un disque de sol (Goyal et al, 1982 ; Raats, 1985), nous fabriquons des disques en étalant la pâte sur des plaques de plexiglass à l'intérieur d'anneaux de $60 \mathrm{~mm}$ de diamètre (compatible avec les dimensions des plantules) et d'épaisseurs comprises entre 1,3 et $5,5 \mathrm{~mm}$ (sensiblement du domaine des croûtes structurales (Boiffin, 1984)). Dans ces conditions la théorie de la flexion des plaques circu- laires de Timoshenko et Woinowsky-Krieger (1961) pourra être appliquée. Les croûtes sont amenées progressivement à des teneurs en eau connues (entre 24,8 et $7,3 \%$ ) dans une enceinte climatique à $20^{\circ} \mathrm{C}$.

\section{Méthodes}

Les expériences sont réalisées dans une enceinte climatique à l'obscurité, à $10^{\circ} \mathrm{C}, 15^{\circ} \mathrm{C}$ et $20^{\circ} \mathrm{C}$ pour les déterminations de forces et seulement à $20^{\circ} \mathrm{C}$ pour les expérimentations d'émergence sous croûtes.

\section{Dispositif de culture}

Les graines germées sont mises en croissance jusqu'à un âge connu depuis la germination et correspondant à un stade précis de la croissance souterraine. Les plantules obtenues sont utilisées pour les mesures de force et pour l'étude de l'émergence. Pour la mise en croissance des plantules, pour les mesures de forces de croissance et pour le suivi de l'émergence sous des obstacles, le dispositif est le même et correspond à celui mis au point pour l'étude de la croissance souterraine des plantules de carotte (Tamet, 1992). Les graines germées ou les plantules sélectionnées sont transplantées dans des pots de culture contenant du sable de Fontainebleau (granulométrie : 150 à $210 \mu \mathrm{m}$ et densité apparente : 1,43). L'humidité pondérale de ce milieu de culture a été définie de façon à éviter les risques de stress hydrique et d'anoxie pour les plantules ; ainsi une humidité de $20 \%$ jugée non limitante pour la germination de la betterave sucrière (Richard, 1988) représente, après vérification pour la carotte, un compromis permettant de satisfaire les besoins en eau et en oxygène de plantules (potentiel hydrique : $<2$ $\mathrm{KPa}$ et porosité libre à l'air : $17 \%$ ). L'alimentation minérale des plantules est assurée grâce à une solution nutritive adaptée à la croissance des jeunes plantules (Saglio et Pradet, 1980) concentrée 3 fois (Tamet, 1992). Cette solution est apportée à l'humectation du sable lors de la transplantation. Par la suite, l'humidité du milieu de culture, contrôlée par pesée des pots, est maintenue constante par apport d'eau déminéralisée tous les $2 \mathrm{j}$.

\section{Mesure de la force de croissance}

Le dispositif mis au point au laboratoire a déjà été utilisé pour des mesures de forces de croissance de racines et de parties aériennes d'autres espèces (Souty, 1987 ; Souty et Stepniewski, 1988; Souty et al, 1992 ; Souty et Rode, 1993). II est basé sur la déformation d'une lamelle d'acier montée en poutre double appui, sous l'action de la force exercée par la plantule (fig 1). Cette déformation est mesurée à l'aide d'une jauge extensométrique (résistance) collée au milieu de la lamelle d'acier. Pour un milieu élastique, non pénétrable il existe en mécanique une relation linéaire entre la force et la déformation relative $\Delta \mathrm{a} / \mathrm{a}: F=\alpha^{*}(\Delta \mathrm{a} / \mathrm{a})$; 


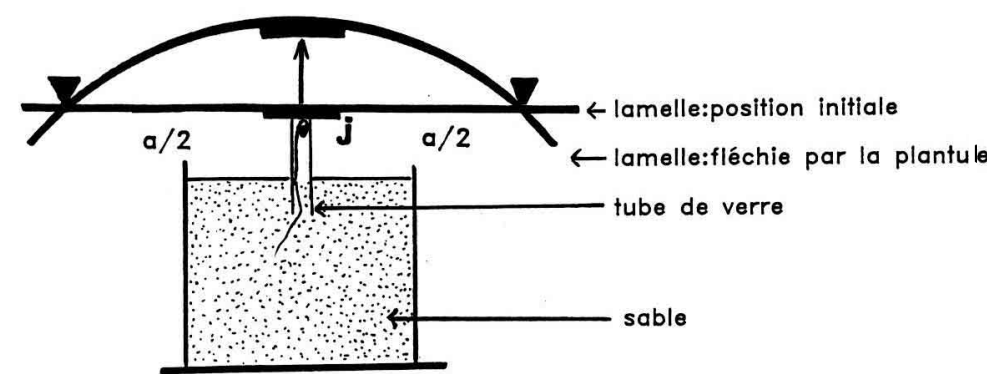

a:portée de la lamelle

J:jauge extensométrique

Fig 1. Dispositif de mesure de la force d'émergence des plantules.

pour notre dispositif le coefficient $\alpha$ est déterminé par un étalonnage préalable de la lamelle.

La partie supérieure de l'hypocotyle de la plantule est insérée dans un tube de verre servant de guide pour maintenir vertical l'effort exercé par la plantule. Chaque pot contenant une plantule est solidement fixé sous une lamelle : le sommet de l'hypocotyle est placé au milieu de la lamelle et effleure la jauge. Les temps de mesure (repérés à partir de la mise en place sous la lamelle) ont été choisis d'après les résultats obtenus lors de l'étude de la croissance souterraine des plantules, soit $24 \mathrm{~h}$ à $20^{\circ} \mathrm{C}, 36 \mathrm{~h}$ à $15^{\circ} \mathrm{C}$ et $48 \mathrm{~h}$ à $10^{\circ} \mathrm{C}$ (Tamet, 1992).

La description des différents traitements expérimentaux réalisés apparaît sur le tableau I. Pour chaque traitement, 5 séries de 10 plantules ont été mesurées. La saisie des déformations de la lamelle à intervalle de temps réguliers donne la possibilité de suivre l'action de la plantule pendant la durée de l'expérimentation. Dans ces conditions, certains paramètres de la croissance de la plantule peuvent être déterminés : d'une part $T_{0}(\mathrm{mn})$, temps de latence au bout duquel la plantule commence à exercer un effort et $T_{\max }(\min )$ temps au bout duquel se produit la déformation maximale de la lamelle $\varepsilon_{\max }$, d'autre part $F_{h}$ la force exprimée par $\alpha{ }^{*}\left(\varepsilon_{0}-\varepsilon_{\max }\right)$ avec $\varepsilon_{0}$ : déformation de la lamelle à la

Tableau I. Traitements expérimentaux pour la caractérisation de la force d'émergence des plantules.

Poids Temps Temps depuis la germination semences $(C)$

Fin phase 1 Mi-phase 2 Fin phase 2

$\begin{array}{lrrlc}\text { GS } & 20 & 4 j & 6 j & 8 j \\ \text { PS } & 20 & 4 j & - & 8 j \\ \text { GS } & 15 & 6 j & - & 11,5 j \\ \text { GS } & 10 & 10 j & - & 20 j\end{array}$

GS $=$ grosses semences $;$ PS = petites semences.

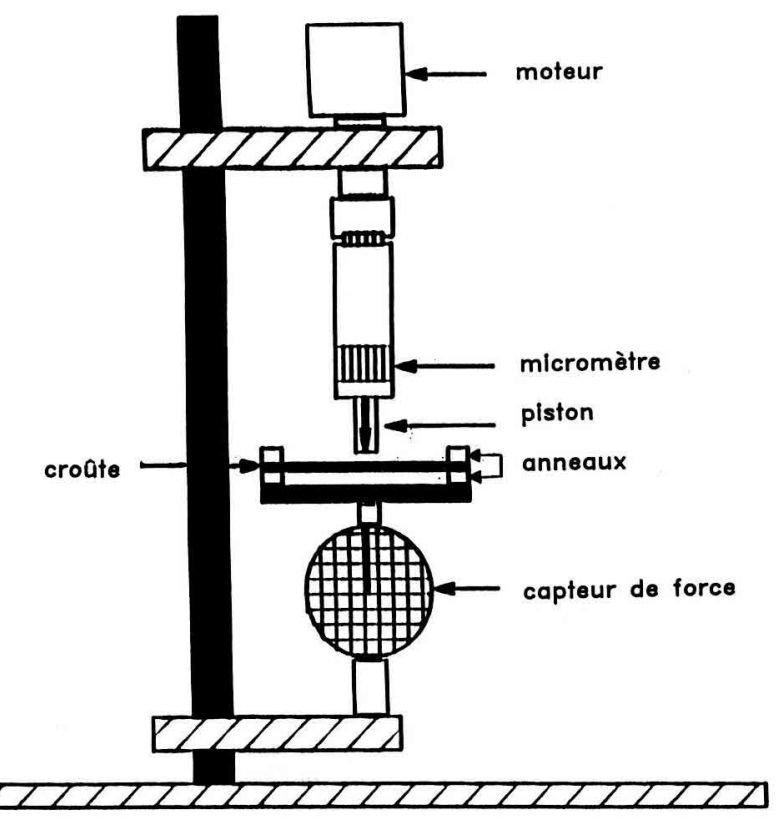

Fig 2. Dispositif pour la mesure de la résistance d'une croûte.

mise en place et la durée de la déformation de la lamelle par $T_{\max }-T_{0}$.

\section{Caractérisation des croûtes superficielles}

La résistance $F_{e}$ d'un lot de croûtes de sol de la Manche est mesurée à l'aide d'un pénétromètre mis au

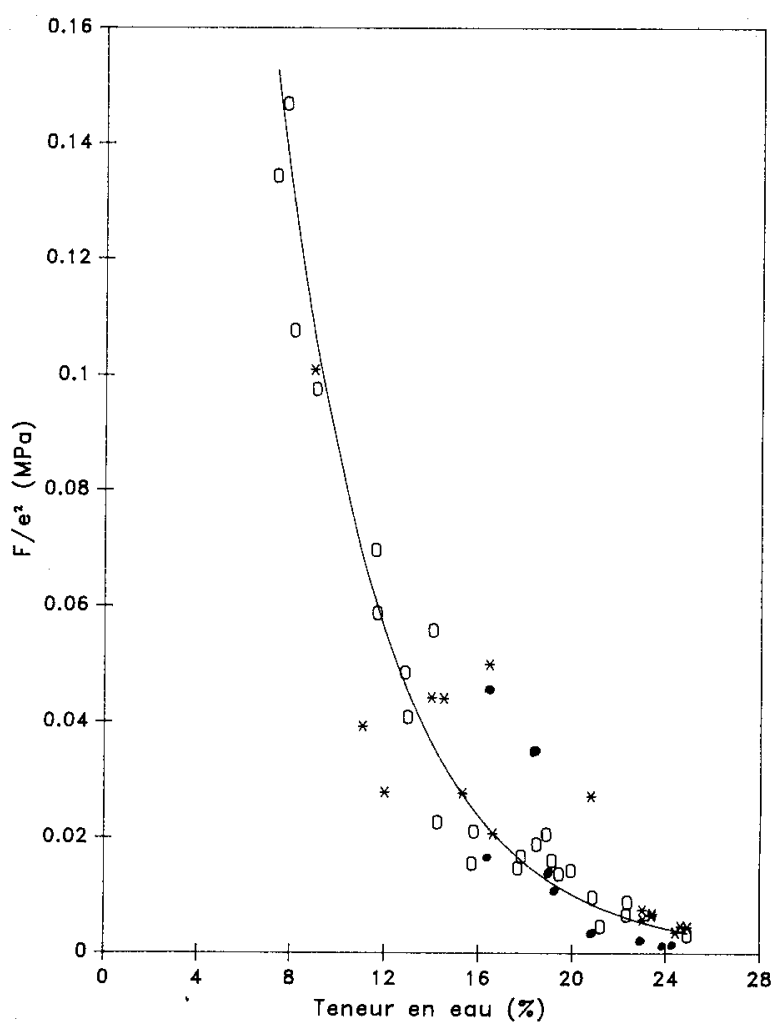

Fig 3. Module de rupture pour les croûtes fabriquées à partir du sol de la Manche. $(\bullet)$ croûtes $5 \mathrm{~mm}$; (o) croûtes $3 \mathrm{~mm}$; (*) croûtes $2 \mathrm{~mm}$. 
point par Guérif (1988) et légèrement modifié pour ces mesures (fig 2) : elle varie avec la teneur en eau pondérale $W$ et l'épaisseur e. II est alors possible d'exprimer, en fonction de la teneur en eau, la variation du paramètre «module de rupture»: $F / e^{2}$, souvent utilisé en mécanique pour caractériser un matériau (ici un sol) : il diminue quand la teneur en eau augmente (fig 3). Le meilleur ajustement (exponentiel) :

$$
\begin{gathered}
F / e^{2}=0,736{ }^{*} \operatorname{EXP}\left(-0,215^{*} W\right) \\
r^{2}=0,8466
\end{gathered}
$$

permet de calculer la résistance de toute croûte de teneur en eau et d'épaisseur connues, utilisées pour des expérimentations d'émergence.

\section{Suivi de l'émergence}

Le dispositif expérimental adopté est mécaniquement identique à celui utilisé pour la mesure des forces de croissance et pour la caractérisation des croûtes. Des plantules de $4 \mathrm{j}$ (depuis la germination) issues de grosses semences et de petites semences sont préparées comme pour la mesure de la force. Les expérimentations d'émergence sur plantules provenant de grosses semences ont été réalisées sur un grand nombre d'individus (184) pour tester la validité d'une part de la prise en compte de la variabilité de la force de croissance et d'autre part du processus de rupture par flexion. II a paru ensuite intéressant de tester l'effet du poids des semences sur le taux d'émergence : pour cela des essais pour seulement quelques valeurs de résistance ont été réalisés sur de petites semences (47). Parallèlement des croûtes (231) d'épaisseurs comprises entre 1,9 et $5,9 \mathrm{~mm}$ et de 10 à $28 \%$ de teneur en eau pondérale ont été fabriquées avec le sol de la Manche selon le procédé décrit précédemment. Leur résistance est calculée à partir de la relation [1] précédemment établie pour le sol considéré. Ces croûtes sont posées sur les pots, séparées du sable par une feuille d'aluminium (percée en son centre pour permettre le passage de l'hypocotyle en croissance) empêchant une modification de la teneur en eau de la croûte; la crosse de l'hypocotyle affleure la partie inférieure de la croûte maintenue par un anneau (fig 4). La mise en place d'un anneau qui maintient la croûte de façon rigide à la surface du pot permet de simuler une forte adhésion de la croûte superficielle avec la sous-couche ; ainsi la plantule doit impérativement rompre la surface pour émerger. La surface des pots est observée pendant environ $4 \mathrm{j}$ et chaque apparition d'une plantule à travers une

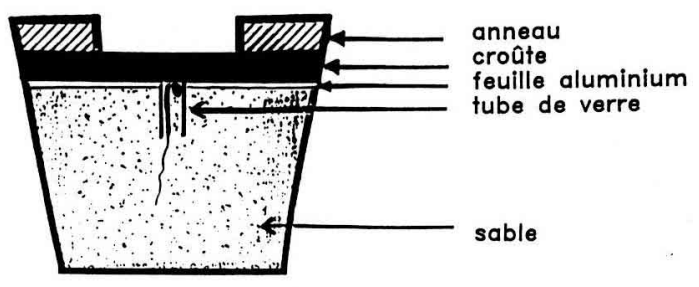

Fig 4. Montage pour l'étude expérimentale de l'émergence sous obstacle. croûte est notée dans le temps. Des groupes de pots de culture sont formés en rassemblant ceux dont les croûtes ont des résistances de valeurs proches : les taux d'émergence sont alors calculés pour chaque groupe correspondant à une résistance moyenne connue.

\section{Calcul théorique de l'émergence}

L'hypothèse d'une rupture par flexion de la croûte sous l'action de la force de la plantule a été validée dans le cas de l'hypocotyle de betterave (Souty et Rode, 1993).

Certaines hypothèses sur la géométrie et les propriétés des croûtes, leurs dimensions par rapport à I'hypocotyle sont faites : les croûtes sont isotropes et élastiques, l'épaisseur de la croûte est petite par rapport à son diamètre, la croûte est grande comparativement aux dimensions de la plantule. Dans ces conditions la théorie des plaques minces de Timoshenko et Woinowsky-Krieger (1961) peut être appliquée et permet de calculer la contrainte maximale en traction apparaissant au centre de la face inférieure de la croûte sous l'action d'une charge uniformément répartie sur une surface connue. Pour un cercle (cas de l'embout du pénétromètre) elle s'écrit :

$\Gamma_{\max }=\left(F / e^{2}\right) *(1+v)^{*}(3 / 2 \pi) *\left(1 / 4(r a)^{2}-\log _{e}(r / a)\right)[2]$

avec $a$ : demi-portée de la plaque circulaire $=22$ * $10^{-3}$ $\mathrm{m} ; \boldsymbol{e}$ : épaisseur de la plaque $(\mathrm{m}) ; r$ : rayon de la charge $=3^{*} 10^{-3} \mathrm{~m} ; v$ : coefficient de Poisson $; F$ : force appliquée par la charge $(\mathrm{N})$.

Dans le cas de la crosse de I'hypocotyle de carotte, la force est supposée être répartie sur une surface elliptique ; alors l'expression de la contrainte maximale est :

$$
\begin{aligned}
\Gamma_{\max } & =\left(F / e^{2}\right) *(1+v)^{*}(3 / 2 \pi) *\left(1 / 4\left(\sqrt{ } b^{*} \sqrt{ } h / 2 a\right)^{2}\right. \\
& \left.-\log _{e}\left(\sqrt{ } b^{*} \sqrt{ } h / 2 a\right)\right)
\end{aligned}
$$

où $b$ et $h$ sont les axes de l'ellipse mesurés sur des plantules de $4 \mathrm{j}$ à $20^{\circ} \mathrm{C}: b=0,70 \mathrm{~mm}$, écart type $=$ $0,148 \mathrm{~mm}$ et $h=1,45 \mathrm{~mm}$, écart type $=0,205 \mathrm{~mm}$.

Pour rompre une même croûte, l'hypocotyle et l'embout du pénétromètre donnent naissance à une même contrainte maximale en traction ; donc en égalisant les expressions [2] et [3] :

$$
F(\text { embout })=1,4{ }^{*} F(\text { hypocotyle })
$$

Cette relation permet de savoir si une plantule est capable de franchir un obstacle donné. Par exemple une plantule dont la force mesurée à l'aide du dispositif lamelle est $F_{h}$ doit pouvoir franchir selon un processus de rupture par flexion toute croûte dont la résistance mesurée avec le pénétromètre ou calculée à partir de l'équation de régression [1] est inférieure à $1,4^{\star} F_{h}$. Réciproquement une croûte de résistance $F_{e}$ peut être rompue par toute plantule dont la force mesurée est supérieure à $F_{e} / 1.4$. 


\section{RÉSULTATS ET DISCUSSION}

\section{Caractéristiques de croissance des plantules}

Sur l'ensemble des traitements les valeurs mesurées de ces caractéristiques sont très variables: de 0 à $48 \mathrm{~g}$ pour les forces, de 0 à $1100 \mathrm{~min}$ pour le temps de latence et de 36 à $2774 \mathrm{~min}$ pour la durée d'effort de la plantule jusqu'à sa force maximale. Les valeurs moyennes de ces caractéristiques pour les différents traitements expérimentaux sont rassemblées dans le tableau IIa. Nous avons choisi de tester l'influence du temps écoulé depuis la germination (âge), du poids des semences, de la température sur ces caractéristiques de croissance et pour cela avons comparé les traitements 2 à 2 après avoir effectué une transformation des variables de façon à obtenir des distributions de valeurs proches d'une loi normale (pour les forces : $(F)^{1 / 2}$ et pour le temps de latence et la durée d'effort $\left(T_{0}\right)^{1 / 4}$ et (Durée $)^{1 / 4}$ ). Dans le tableau Ilb figurent, pour chaque comparaison, les niveaux de signification de la condition de rejet de l'hypothèse nulle (test de Student).

\section{Effet de l'âge des plantules}

Nous constatons que la force moyenne développée à $20^{\circ} \mathrm{C}$ par des plantules de $4 \mathrm{j}$ depuis la germination, issues de grosses semences, est de $9,8 \mathrm{~g}$ : elle est significativement supérieure aux forces moyennes des plantules de 6 et $8 \mathrm{j}$ ( 3 et 4 g) qui en revanche ne diffèrent pas (tableau llb). Ces mêmes plantules de $4 \mathrm{j}$ ont des temps de latence et des durées de développement de force significativement supérieurs aux valeurs correspondantes des plantules plus âgées (sauf pour le temps de latence des plantules de $6 \mathrm{j}$ ).

Tableau Ila. Valeurs moyennes des caractéristiques des plantules pour les différents traitements expérimentaux.

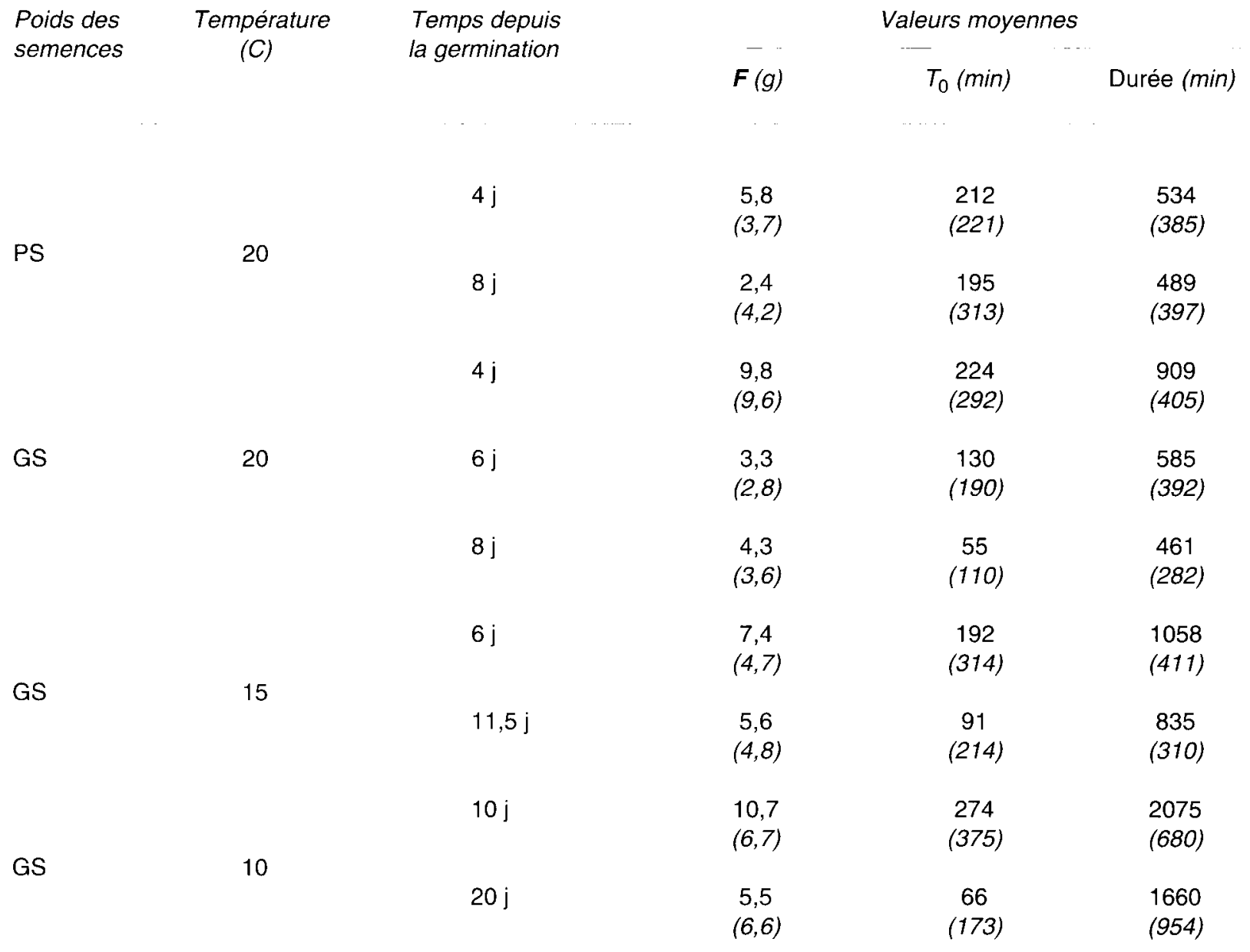


La force de croissance des plantules dépend de la pression de turgescence et des capacités d'ajustement osmotique des cellules par accumulation de réserves ou par absorption d'éléments minéraux (Hettiaratchi et O'Callaghan, 1974 ; Kutschera, 1991 ; Meyer et Boyer, 1981). Ceci peut être rapproché de la diminution de la force de croissance observée en fin de deuxième phase, car, à ce stade, l'élongation de l'hypocotyle, et surtout sa croissance pondérale, s'achèvent avec l'arrêt de transfert des réserves en provenance des cotylédons. Toutefois la force de croissance n'est pas nulle et elle diminue très fortement dès le début de la deuxième

Tableau Ilb. Comparaison des valeurs moyennes des caractéristiques des plantules. Niveau de signification du rejet de l'hypothèse nulle pour: F, T0 et Durée.

$\begin{array}{lll} & & 20^{\circ} \mathrm{C} \\ P S & P S & G S \\ 4 J & 8 J & 4 J \\ & & \\ & & \\ & & \\ & & \\ & & \\ & & \\ & & \\ & 0,4 & 0,1 \\ 0,7 & 0,9 \\ & & \end{array}$

PS 8J $\quad 0,001$

0,4

0,7

GS 4J

0,1

0,9

0,001

0,001

GS 6J

0,001

0,4

0,003

0,001
0,4

0,003

GS GS

GS

\begin{tabular}{lr}
\multicolumn{2}{c}{$15^{\circ} \mathrm{C}$} \\
GS & GS \\
$6 \mathrm{~J}$ & $11,5 \mathrm{~J}$
\end{tabular}

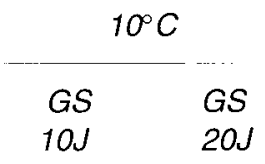

GS 8J

$\begin{array}{lll}\mathbf{0 , 0 0 3} & \mathbf{0 , 0 1} & \mathbf{0 , 2} \\ 0,7 & 0,06 & 0,3 \\ 0,9 & 0,003 & 0,3\end{array}$

$15^{\circ} \mathrm{C}$

GS $6 \mathrm{~J}$

0,6

0,3

0,2

GS $11,5 \mathrm{~J}$

0,6

0,7

0,7

0,9

0,003

0,01

0,6

0,5

0,3

0,9

0,003

0,2

0,001

0,2

0,3

0,3

\begin{tabular}{|c|c|c|c|c|}
\hline & & $\begin{array}{l}0,7 \\
0,001\end{array}$ & & \\
\hline $10^{\circ} \mathrm{C}$ & & & & \\
\hline GS $10 \mathrm{~J}$ & $\begin{array}{l}\mathbf{0 , 5} \\
0,9 \\
0,001\end{array}$ & & $\begin{array}{l}\mathbf{0 , 2} \\
0,4 \\
0,001\end{array}$ & \\
\hline GS $20 \mathrm{~J}$ & & $\begin{array}{l}0,9 \\
0,4 \\
0,001\end{array}$ & & $\begin{array}{l}\mathbf{0 , 7} \\
0,8 \\
0,06\end{array}$ \\
\hline
\end{tabular}

0,2

0,4 0,001
0,9

0,4

0,001
0,7

0,8

0,06 
phase alors que I'hypocotyle poursuit sa croissance.

La variation de la valeur de la force de croissance au cours de la croissance souterraine n'a pas fait l'objet de travaux dans la bibliographie étudiée.

\section{Effet du poids de la semence}

Les plantules de 4 et $8 j$, issues de petites semences, exercent respectivement des forces moyennes de 5,8 et $2,4 \mathrm{~g}$ (tableau lla) : seules les plantules de $8 \mathrm{j}$ ont des forces significativement inférieures à celles exercées par les plantules de même âge mais issues de grosses semences.

Cet effet du poids des semences a été observé par d'autres auteurs. Ainsi Williams (1956) montre que la variabilité du poids des semences de 4 légumineuses fourragères expliquerait $68 \%$ de la variabilité des forces exercées. II trouve une corrélation élevée avec la masse de carbone présente dans la semence $\left(r^{2}=0,84\right)$. On peut penser que, dans le cas de petites semences, la moindre quantité de réserves séminales diminue la possibilité d'ajustement osmotique des cellules de l'hypocotyle.

\section{Effet de la température}

Les forces moyennes exercées aux 3 températures $\left(20,15\right.$ et $\left.10^{\circ} \mathrm{C}\right)$ ne sont pas significativement différentes (tableau IIb). En revanche les temps nécessaires à l'obtention des forces maximales sont significativement différents et augmentent avec l'abaissement de température.

Les variations de température peuvent modifier la perméabilité et l'élasticité des membranes cellulaires (Hettiaratchi et O'Callaghan, 1984). Dans le cas de la carotte des températures de $15^{\circ} \mathrm{C}$ et $10^{\circ} \mathrm{C}$ ne sont peut être pas suffisamment basses pour modifier les caractéristiques des membranes. En effet l'élongation de l'hypocotyle n'est pas diminuée à basse température mais seulement ralentie. Ainsi peut être expliquée l'absence d'effet de la température sur la force, d'autant plus que le temps de mesure tient compte de cette croissance plus lente.

\section{Représentation des forces de croissance}

Nous venons d'établir quels étaient les paramètres influençant les valeurs des forces mais

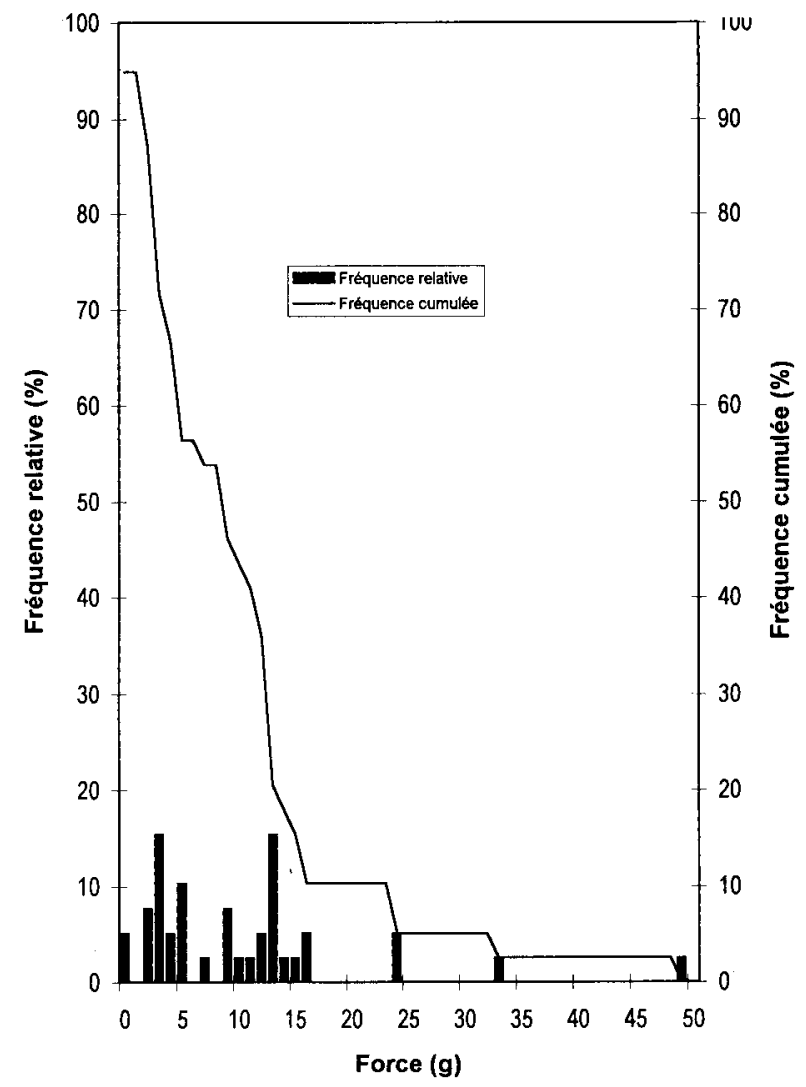

Fig 5. Forces enregistrées et mode d'expression (GS, $20^{\circ} \mathrm{C}$, 4 j depuis germination).

nous pouvons aussi, pour chaque traitement expérimental, considérer la répartition de ces valeurs en calculant leur fréquence relative au nombre total des plantules étudiées (fig 5): chaque barre de l'histogramme représente la proportion de plantules exerçant une force comprise entre 2 valeurs d'abscisse différant de $1 \mathrm{~g}$. Cette variabilité importante mise en évidence, est responsable pour une grande part des différences observées dans les taux d'émergence; la courbe de la figure 5, pour laquelle l'ordonnée de chaque point représente la fréquence cumulée de plantules exerçant une force strictement supérieure à une valeur seuil exprimée par l'abscisse, peut alors constituer une expression de la variation du taux d'émergence. Ce mode de représentation des forces montre que, par exemple dans le cas des plantules à $20^{\circ} \mathrm{C}$ issues de grosses semences, $40 \%$ environ des plantules de $4 \mathrm{j}$ et seulement 4 à $10 \%$ des plantules de 6 et 8 j exercent une force d'au moins $10 \mathrm{~g}$ (fig 6). Par ailleurs les différences de forces exercées se traduisent par des courbes de fréquences cumulées distinctes entre classes de poids de semences (fig 7) : les plantules de $8 \mathrm{j}$, issues de petites semences, se caractérisent par une grande proportion $(50 \%)$ de plantules de 


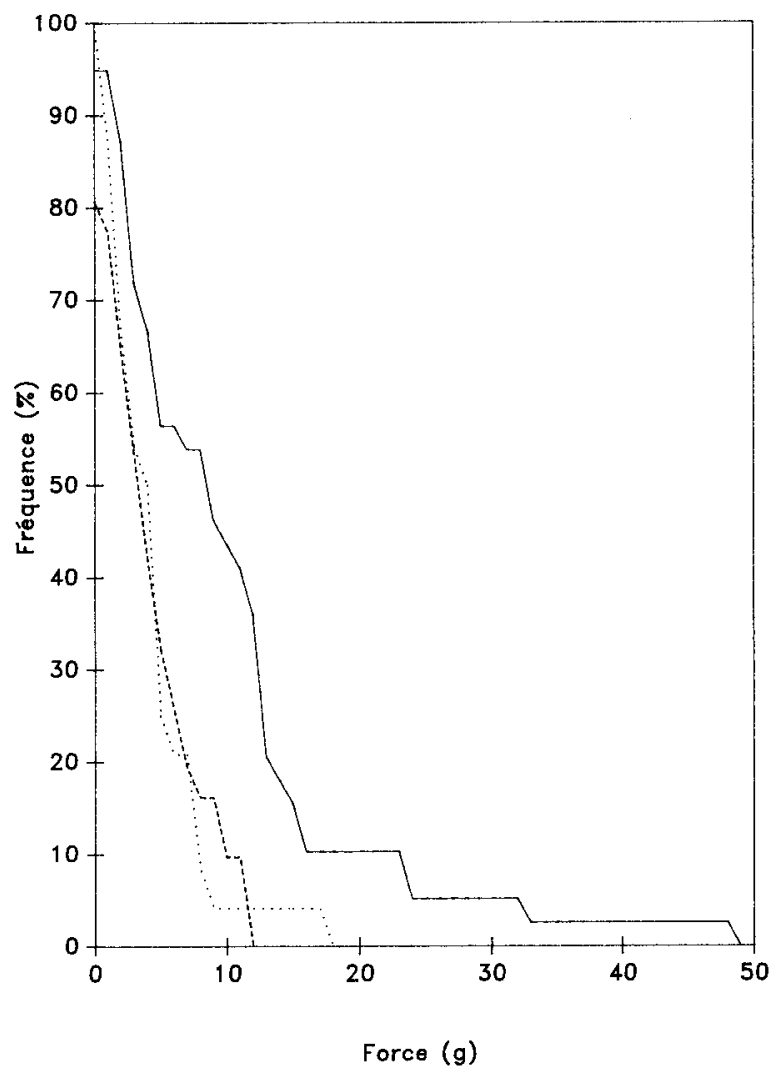

Fig 6. Effet du temps de croissance souterraine sur les forces d'émergence exercées. (-) $4 \mathrm{j} ;(--) 6 \mathrm{j} ;$ (..) $8 \mathrm{j}$ depuis germination, GS, $20^{\circ} \mathrm{C}$ ).

force très faible non détectable par le dispositif de mesure. Même si les forces ont été trouvées non significativement différentes (cas de plantules de $4 \mathrm{j}$ à $20^{\circ} \mathrm{C}$ issues des grosses et des petites semences), les courbes de distribution des fréquences cumulées peuvent être distinctes : ainsi la fréquence des forces supérieures à $10 \mathrm{~g}$ pour les petites semences est très faible par rapport à celle pour les grosses semences (fig 7).

\section{Émergence des plantules}

\section{Émergence théorique}

La relation [4] permet, comme il a été décrit précédemment, de connaître la possibilité d'émergence d'une plantule de force connue à travers une croûte de résistance déterminée. Si le raisonnement découlant de cette relation [4] est appliqué à une population de plantules dont la distribution des forces a été préalablement établie, il est alors possible, par une homothétie de rapport 1,4 de transformer la courbe de fréquence cumulée des forces en une courbe représentant le taux

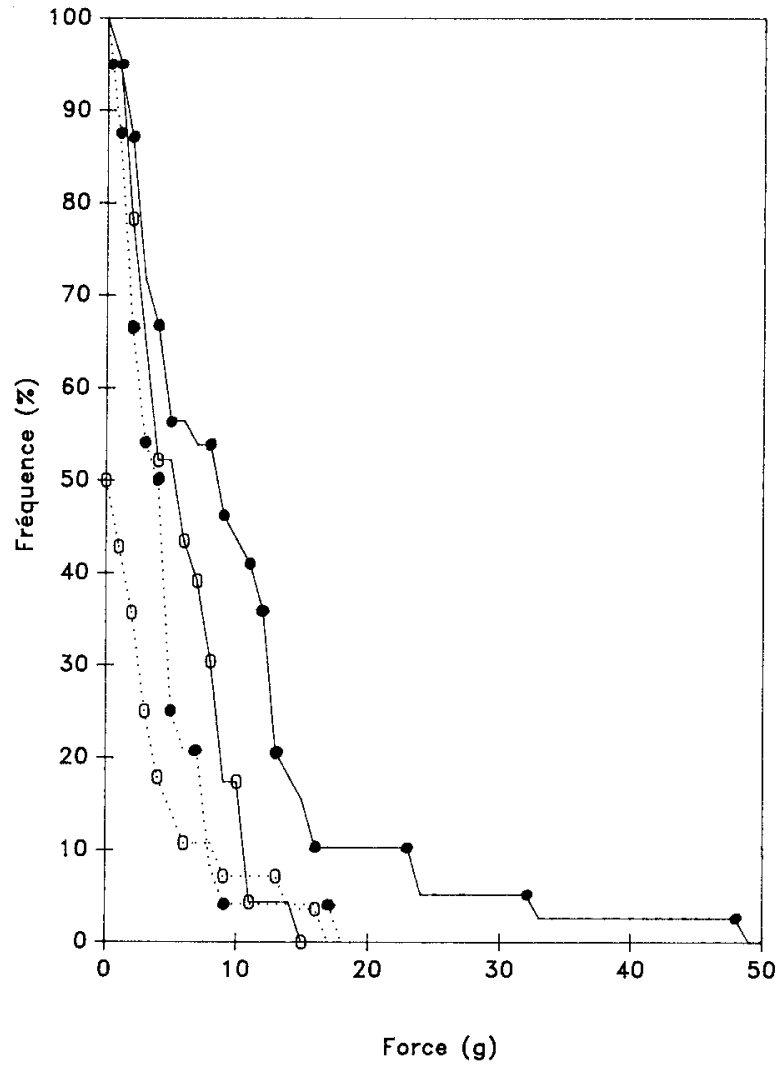

Fig 7. Effet du poids des semences sur les forces d'émergence exercées. (•) GS ; $\left(^{\circ}\right) \mathrm{PS} ;(-) 4 \mathrm{j} ;(\ldots) 8$ j depuis germination à $20^{\circ} \mathrm{C}$.

d'émergence théorique en fonction de la résistance de l'obstacle (fig 8). Cette figure met aussi en évidence que, pour une même résistance de croûte, le pourcentage d'émergence des plantules issues des petites semences est plus faible que celui des plantules issues de grosses semences.

\section{Comparaison émergence expérimentale - émergence théorique}

La figure 8 rassemble aussi les résultats expérimentaux relatifs à l'émergence des plantules issues des grosses semences et des petites semences.

II y a une bonne adéquation globale entre les taux d'émergence observés et les fréquences théoriques calculées à partir du modèle de rupture par flexion (fig 9). Les taux d'émergence diminuent progressivement lorsque la résistance de l'obstacle augmente : ils sont nuls pour une résistance de l'ordre de $0,5 \mathrm{~N}$. Les résultats rendent compte des différences de distributions statistiques des forces observées entre les 2 classes de poids de semences. Pour une résistance de croûtes au plus égale à $0,1 \mathrm{~N}$, les taux d'émergence observés sont identiques. Pour des résis- 

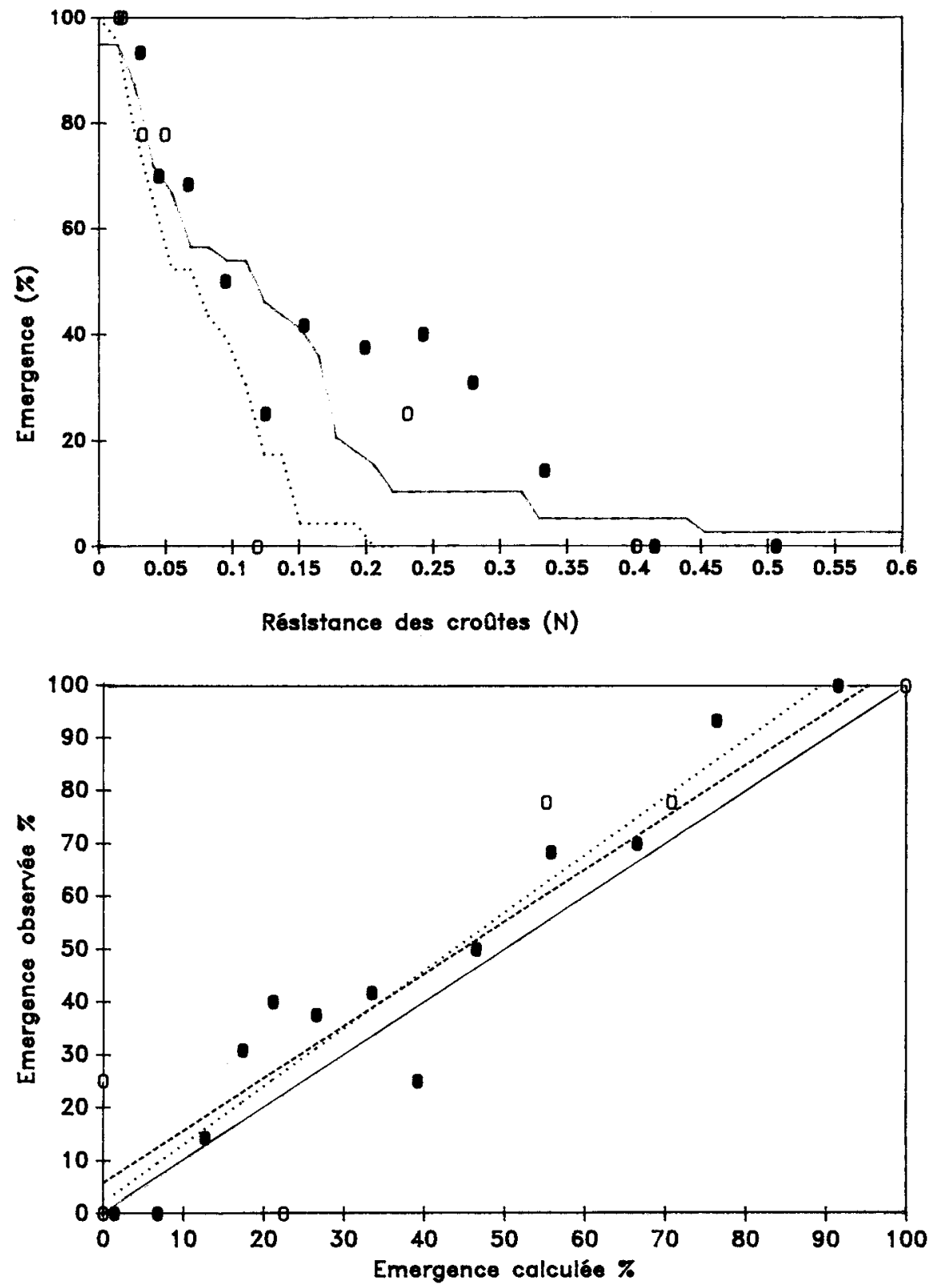

Fig 8. Émergence à $20^{\circ} \mathrm{C}, 4 \mathrm{j}$ après germination. GS : - courbe théorique (modèle flexion) ; - points expérimentaux ; PS : --courbe théorique (modèle flexion) ; ${ }^{\circ}$ points experimentaux.
Fig 9. Comparaison : taux d'émergence observés - taux d'émergence prévus. $(\bullet)$ GS ; $\left(^{\circ}\right)$ PS. tances supérieures, les taux d'émergence des plantules issues de grosses semences sont les plus élevés.

Toutefois les figures 8 et 9 mettent en évidence que les taux d'émergence observés sont souvent supérieurs aux taux calculés, en particulier pour des résistances faibles ou relativement élevées. En revanche, dans le cas d'une observation, pour chaque classe de poids de semences, le taux observé est bien inférieur au taux calculé.

En effet pour des résistances de 0,1 à $0,142 \mathrm{~N}$ dans le cas des petites semences et de 0,115 à $0,135 \mathrm{~N}$ pour les grosses semences, les taux de levée sont inférieurs aux prévisions (dans le cas d'une observation, pour chaque classe de poids de semences, le taux observé est même très inférieur au taux calculé). Pour des résistances de
0,171 à $0,291 \mathrm{~N}$ pour les petites semences et de 0,140 à $0,305 N$ pour les grosses semences, les taux de levée sont supérieurs aux taux calculés.

Dans le premier cas il y a une majorité de croûtes fines et sèches: pour les petites semences les croûtes ont une épaisseur de 2 $\mathrm{mm}$ et des teneurs en eau pondérales de 14 à $16 \%$, pour les grosses semences les croûtes sont de $3 \mathrm{~mm}$ pour une humidité pondérale de 17 à $21 \%$. Dans le deuxième cas pour les petites et les grosses semences il y a une majorité de croûtes d'environ $5 \mathrm{~mm}$ pour des humidités pondérales de 18 à $23 \%$ (figs 10,11).

Ces observations révèlent donc un biais du dispositif expérimental quant à la répartition des épaisseurs de croûtes dans chaque classe de résistance. Par ailleurs les écarts entre valeurs 
Fig 10. Caractéristiques des croûtes fabriquées pour l'étude de l'émergence des plantules issues des GS (les points de la partie supérieure du graphique correspondent aux épaisseurs les plus élevées). (+) les plantules ont levé ; $\left({ }^{\circ}\right)$ les plantules n'ont pas levé.

Fig 11. Caractéristiques des croûtes fabriquées pour l'étude de l'émergence des plantules issues des PS (les points de la partie supérieure du graphique corres. pondent aux épaisseurs les plus élevées). (+) les plantules ont levé $;\left(^{\circ}\right)$ les plantules n'ont pas levé.
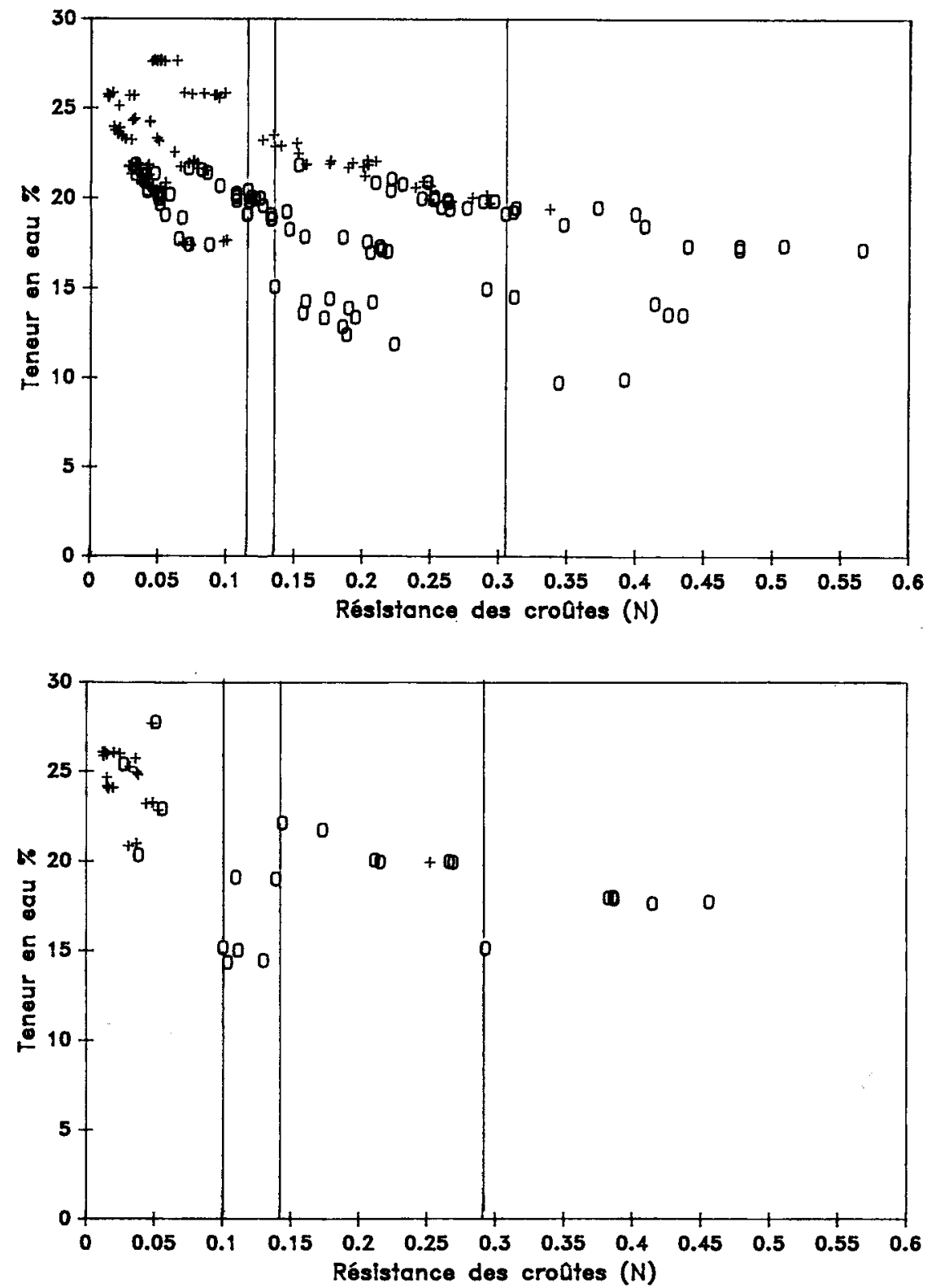

observées et calculées peuvent être rapprochés de la variabilité résiduelle autour du module de rupture (fig 3). En effet dans la gamme de 10 à $20 \%$ d'humidité pondérale, quelle que soit l'épaisseur des croûtes, il existe une variabilité autour du module de rupture qui entraîne une imprécision sur le calcul de la résistance et donc sur la valeur de l'abscisse des points expérimentaux. Dans la gamme de 20 à 25\% d'humidité pondérale la variabilité sur le module de rupture conduit à une surestimation de la résistance pour des croûtes de $5 \mathrm{~mm}$. Dans ces conditions, l'abscisse des points expérimentaux pourrait être plus faible dans la zone où les taux d'émergence calculés sont les plus élevés.

Enfin le passage des plantules à travers des croûtes humides pourrait se faire selon un pro- cessus différent d'une rupture par flexion, peutêtre un enfoncement qui a tendance à donner des taux d'émergence calculés supérieurs à ceux calculés pour une rupture par flexion (le facteur de passage de Fe à Fh est supérieur à 1,4). Mais l'écart entre ces valeurs est trop important pour conclure à un enfoncement seul.

\section{CONCLUSION}

La force de croissance exercée par une plantule de carotte, de 2,4 à $10,9 \mathrm{~g}$ en moyenne, est faible comparée à celle d'autres espèces (tableau III), ce qui tend à expliquer la forte sensibilité de cette culture à l'état structural du lit de semences. La grande variabilité existant dans la 
Tableau III. Forces d'émergence de différentes espèces végétales.

$\begin{array}{ccc}\text { Espèces } & \text { Ordre de grandeur des forces d'émergence mesurées (g) } & \text { Auteur } \\ \text { Coton } & 200 & \begin{array}{c}\text { Garner et Bowen (1966) } \\ \text { Gerard (1980) }\end{array} \\ \text { Maïs } & 200 \text { à } 600 & \begin{array}{c}\text { Prihar et Aggarwal (1975) } \\ \text { Souty et al (1992) }\end{array} \\ \text { Blé } & 100 & \text { Bouaziz (1990) } \\ \text { Betterave sucrière } & 0 \text { à } 200 & \text { Souty (1993) } \\ \text { Luzerne } & 30 & \text { Williams (156) } \\ \text { Trèfle } & 5 \text { à } 10 & \text { Jensen et al (1972) } \\ \text { Trèfle souterrain } & 15 & \text { Jensen et al (1972) } \\ \text { Williams (1956) }\end{array}$

levée peut être attribuée à l'hétérogénéité importante subsistant entre plantules. Cette variabilité apparaît par ailleurs fortement liée à l'état dans lequel se trouvent les plantules au cours de leur croissance souterraine. Les potentialités d'émergence diminuent lorsque la croissance souterraine est prolongée, ce qui met en évidence le rôle important du contrôle de la régularité de la profondeur de semis au champ. Les potentialités d'émergence sont inférieures dans le cas de plantules issues de semences de faible poids. Le choix de semences de poids élevé semble donc être un facteur de diminution des risques d'échec de l'implantation. Les températures basses sont la cause d'un ralentissement du développement de la force mais elles n'affectent pas la grandeur de la force. Toutefois en prolongeant le temps de croissance souterraine, les températures basses augmentent la probabilité d'apparition de conditions plus difficiles lors de la levée, telle que le déssèchement de la croûte superficielle qui devient plus résistante.

Un modèle de prévision du taux d'émergence des plantules a été établi dans des conditions expérimentales bien précises. Ce modèle prend en compte simultanément la distribution statistique des forces d'émergence exercées par les plantules et les caractéristiques physiques et mécaniques de l'obstacle ; les taux d'émergence observés sont en accord avec les distributions des forces des plantules et rendent compte des différences de poids des semences dont les plantules sont issues. Ce travail appelle d'autres études dont certaines, en cours, tendent à compléter ce modèle en introduisant une variation dans le temps de la teneur en eau des croûtes superficielles; dans ces conditions nous recherchons l'évolution des taux d'émergence.

\section{REMERCIEMENTS}

Nous remercions MA Marin-Laflèche de l'unité d'agronomie de Laon-Péronne et MC Bruchou de l'unité de biométrie d'Avignon de leur aide efficace pour le traitement statistique de nos données.

\section{RÉFÉRENCES}

Benjamin LR (1982) Some effects of differing times of seedlings emergence, population density and seed size on root-size variation in carrot populations. J Agric Sci Camb 98, 537-545

Benjamin LR (1987) Variation in plant size and timing of carrot production. Acta Hortic 198, 297-304

Boiffin J (1984) La dégradation structurale des couches superficielles du sol sous l'action des pluies. Thèse docteur-ingénieur, Institut national agronomique Paris-Grignon, $320 \mathrm{p}$

Bouaziz A, Souty N, Hicks D (1990) Emergence force exerted by wheat seedlings. Soil Tillage Res 17 , 211-219

Durr C, Tamet V, Boiffin J (1990) Redistribution of seed reserves during the emergence of carrot seedlings and its influence on subsequent growth. In : Proceedings of the First Congress of the European 
Society of Agronomy (A Scaife, ed), Colmar: ESA, Sect 1, p 43

Garner TM, Bowen HD (1966) Plant mechanisms in seedling emergence. Trans ASAE 650-653

Gerard CJ (1980) Emergence force by cotton seedlings. Agron J 72, 473-476

Goyal MR (1982) Soil crusts vs seedling emergence. Review Agricultural Mechanization in Asia, Africa and Latin America Winter 62-78; article $\mathrm{N}^{\circ} 1290$; Journal of University of Puerto Rico, Rio Piedras (USA)

Goyal MR, Drew LO, Dickson DG (1982) Analytical prediction of seedling emergence force. Trans ASAE 25, 38-41

Guérif $J$ (1988) Détermination de la résistance en traction des agrégats terreux : revue bibliographique et mise au point technique. agronomie 8, 281-288

Hettiaratchi DRP, O'Callaghan JR (1974) A membrane model of plant cell extension. $J$ Theor Biol 45,459 465

ISTA (1985) Règles internationales pour les essais de semences, Vol 13, suppl 2, Zurich

Jensen EH, Frelich JR, Gifford RO (1972) Emergence force of forage seedlings. Agron J 64, 635-639

Kutschera $O$ (1991) Osmotic relations during elongation growth in hypocotyls of Helinthus annuus $\mathrm{L}$. Planta 184, 61-66

Meyer RF, Boyer JS (1981) Osmoregulation, solute distribution and growth in soybean seedlings having low water potentials. Planta 151, 482-489

Nicoullaud B (1988) Influence du type de sol sur le comportement d'une culture de carottes. Sci Sol 26, $65-80$

Prihar SS, Aggarwal GC (1975) A new technique for measuring emergence force of seedlings and some laboratory and field studies with corn (Zea mays L). Soil Sci 120, 200-204

Raats PAC (1985) Kinematics and dynamics of seedlings emergence. In : International Symposium on the assessment of soil surface sealing and crusting Ghent, Belgium, 252-261
Richard G (1988) La germination des semences de betterave sucrière (Beta vulgaris $L$ ) en conditions d'hypoxie : modélisation, contribution à un diagnostic au champ. Thèse doct ing, INA-PG, Paris, $102 p$ + annexes

Saglio P, Pradet A (1980) Soluble sugars, respiration and energy charge during aging of excised maize root tipes. Plant Physiol 66, 516-519

Salter PJ, Currah IE, Fellows JR (1980) Further studies on the effects of plant density, spacial arrangement and time of havest on yield and root size in carrots. J Agric Sci Camb 94, 365-378

Salter PJ, Currah IE, Fellows JR (1981) Studies of some sources of variation in carrot root weight. J Agric Sci Camb 96, 549-556

Souty N (1987) Aspect mécanique de la croissance des racines. I. Mesure de la force de pénétration. agronomie 7, 623-630

Souty N, Stepniewski W (1988) The influence of external oxygen concentration on axial root growth force of maize radicles. agronomie $8,295-300$

Souty N, Stengel P, Rode C, Tuttobene R (1992) A mechanistic study of maize emergence through superficial crusts. Soil Tillage Res 23, 125-140

Souty N, Rode C (1993) Emergence of sugar beet seedlings from under different obstacles. Eur $J$ Agron 2, 213-221

Tamet V (1992) Étude de la croissance hétérotrophe des plantules de carotte (Daucus carota $L$ ). Conséquences sur l'implantation de la culture. Thèse doct ing, INA-PG, Paris, $87 \mathrm{p}+$ annexes

Timoshenko S, Woinowsky-Krieger S (1961) Flexion symétrique des plaques circulaires. In : Théorie des plaques et des coques, Librairie polytechnique $\mathrm{C}$ Béranger, Paris, 51-78

Villeneuve F, Leteinturier J (1992) La carotte. Il. État des connaissances. CTIFL ed, Paris, $228 p$

Williams WA (1956) Evaluation of the emergence force exerted by seedlings of small seeded legumes using probit analysis. Agron J 48, 273-274 